\title{
Motivations and Perceptions of Student Service Participation
}

\author{
Lily Elizabeth Johnson a and Amanda Reinke ${ }^{\mathrm{a}}$
}

Researchers studying higher education frequently associated community service with liberal arts education. Most research on service participation among college students predominantly studies mandatory service-learning programs. Several studies gathered survey data to analyze opinions and feelings towards volunteering from students at universities with a service-learning component and found that students who participate feel more connected to and engaged in their community. Georgia College \& State University (Georgia College or GC), a public liberal arts university, also claims community service is an integral part of their mission because it broadens students' perspectives and forms engaged citizens. However, there is little data examining student motivations and perceptions of their voluntary service experiences as part of their education at liberal arts institutions. This research project addresses this gap using two methods: (a) participant observation during service events and (b) semi-structured interviewing with both students engaged in voluntary service activities as well as employees who observe student service involvement. Preliminary findings display a wide array of intrinsic and extrinsic motivations for student service participation, and interview data relates service to the liberal arts mission as a method of expanding perspectives of students. These results support literature that says students benefit from service participation by both honing their skills and spurring their passion about community involvement. The findings herein add to the literature by exploring motivators for voluntary service participation.

Keywords: community service, liberal arts, college students

Community service is consistently listed as a cornerstone of liberal arts education by researchers studying higher education. However, service participation is dynamic and highly variable; it may be sustainable or episodic and differs based on the individual's motivation to participate. Service may be encouraged as either co-curricular, required by a specific class, major, or other educational purpose, or extracurricular, promoted by volunteer organizations not associated with course requirements and requiring a person to decide to volunteer based on their own motivation. The research on service participation predominantly studies mandatory service-learning, particularly where it is cocurricular. Several studies gathered quantitative data with surveys to analyze opinions and feelings towards volunteering from either current students or alumni which portray positive experiences regarding service (Jones, Blinkhorn, Schumann \& Reddy, 2014; White-Farnham \& Meyer, 2014; Fenzel \& Peyrot, 2005). Additionally, a study by McCunny (2017) utilized in-person interviews, as opposed to quantitative research methods, to provide an avenue for in-depth analyses of community involvement. Other resources discuss theories behind participation in community service or the transformation of students' learning in addition to their greater feeling of responsibility achieved through community and civic engagement (Sherman, 2016; Heffner, Curry \& Beverslui, 2006; McCunny, 2017; Dote, Cramer, Dietz \& Grimm, 2006). In keeping with suggested best practices from the Association of American Colleges \& Universities (2019), Georgia College \& State University (Georgia College or GC) promotes community service as a form of experiential learning. Such activities spur engagement with community members and have the potential to widen students' experience and comfort zones. In fact, GC (2019) describes a liberal arts education as a "general, overall education" that "teaches you how to critically assess the world around you," and community service/engagement is frequently referenced as a key to that development. GC supports community service through the GIVE Center, an office on campus that matches students with community service organizations, promotes service events, and attempts to prepare students for their future careers and their lives as engaged citizens. They promote service participation by encouraging volunteerism, leadership, and collaboration, both with student-led service organizations and nonprofits in the local community.

Community service is divided into two categories: sustainable service, which involves volunteering consistently to fulfill a long-term need and provide long-term benefits to the community, and episodic service, which consists of volunteering with a single event or on a non-consistent basis to benefit a group. Although sustainable service more effectively fulfills long-term needs and provides service experiences that are likely to have more extensive positive effects on students and the community, episodic service continues to be a popular volunteer option for many students at $\mathrm{GC}$, reflecting the national average (Dote et al., 2006). Despite the importance of service as part of the universities' principles, there is little data examining student motivations and perceptions of their service experiences as part of their wider education at liberal arts institutions.

There is no clear consensus among researchers that explains why students participate in service. Emmanuel Levinas claims that people are motivated to serve due to an unconscious sense of guilt or responsibility towards the "others"; Robert Rhoads argues that motivation to serve stems from the caring relationships built between the volunteers and recipients of service (Sherman, 2016). However, Dote and other researchers (2006) believe students may volunteer to improve their resume or simply because the opportunity is present. Despite different theories, there is a common motivational thread throughout the literature: students who want to see changes to social injustices volunteer (Sherman, 2016; Heffner et al., 2006; McCunny, 2017). Several studies found that, as a result of participating in service, students developed an increased awareness of social issues and a greater commitment to the underserved in their community (Jones et al., 2014; White-Farnham \& Meyer, 2014; Fenzel \& Peyrot, 2005). These findings are valuable, but the answer to what 
motivates individual students to voluntarily participate in service is still lacking greater detail.

Required service-learning is the main focus of current research on the topic of student volunteerism. There is substantial evidence strengthening advocacy for a service component in college education, especially in relation to sustainable service (Fenzel \& Peyrot, 2005; Jones et al., 2014; White-Farnham \& Meyer, 2014). For example, Fenzel and Peyrot (2005) found that co-curricular service predicted postgraduation service involvement, service-oriented careers, and political activism for alumni in their study. The bridge between college courses and sustainable practices benefitting the greater community is the key to fostering engaged scholarship and more dynamic citizens (Sherman, 2016; Heffner et al., 2006). However, an extension of the current research is needed to study student volunteer perceptions of how extracurricular service interacts with their holistic college experience.

Community engagement and civic responsibility are frequently incorporated into the missions of institutions to showcase their positive values. This is a main reason why service-learning is so prominent in many colleges and universities (McCunny, 2017; Dote et al., 2006). In fact, percentages of college students that volunteer are rising as school missions become more oriented towards service, whether sustainable or episodic (Dote et al., 2006). As seen in McCunny's (2017) study, student's sustainable involvement is

\section{Results}

\section{Motivations for Service Participation}

Perceived students' motivations for service participation vary in the language used by both students and staff in the study, but many of the core principles are the same. Based on themes found in the literature, students are motivated by any number of internally driven factors, such as wanting to help their community (Heffner et al., 2006; McCunny, 2017), and externally driven factors, such as fulfilling a course requirement (Jones et al., 2014; White-Farnham \& Meyer, 2014; Fenzel \& Peyrot, 2005); these are considered intrinsic and extrinsic, respectively, while other motivational factors are not explicitly categorized.

Motivations that are clearly intrinsic include wanting to help others and make a difference in their lives, having a passion about a cause that will better the world, and making other people happy. Other motivations are difficult to classify as either intrinsic or extrinsic; these include a felt obligation or guilt towards a certain group or humanity, wanting to gain future career experiences or different experiences that will expand an individual's perspective and add to their resume, engaging with both other students and service recipients to understand each other better, and having the easy opportunity to engage while earning trackable service hours. The only truly extrinsic motivator found in this research is meeting a class

Table 1. Motivations for Service Participation

\begin{tabular}{|l|l|l|}
\hline Motivator & Total frequency & Transcript frequency \\
\hline Helping others & 48 & 15 \\
\hline Social engagement & 42 & 15 \\
\hline Passion & 33 & 7 \\
\hline New/different experiences & 30 & 12 \\
\hline Class/career experience & 24 & 10 \\
\hline "Values" & 24 & 1 \\
\hline Easy opportunity & 14 & 8 \\
\hline Obligation/guilt & 8 & 6 \\
\hline Happiness of others & 4 & 4 \\
\hline "Intrinsic" & 4 & 2 \\
\hline
\end{tabular}

associated with internal "transformation," though episodic volunteering is becoming a more ideal route of involvement for students who have other commitments (Dote et al., 2006).

These findings, however valuable, produce further questions regarding possible differences in motivation to participate in each type of service and whether or not this involvement has any relation to the institution's mission. To address this gap, two research questions were posed: What motivates GC students to participate in episodic and/or sustainable service? How does participation in community service impact a student's time at GC? In an attempt to answer these questions, both students' motivations to participate in episodic and/or sustainable voluntary service and the perceived relationship between service and a liberal arts education were investigated. The researcher used participant observation to study student participation at GC service events and semistructured interviewing to gain a deeper perspective into students' motivations for volunteering and their perceptions of volunteering as part of their liberal arts education. requirement. This motivator was only mentioned during discussions of participants' initial service experiences. This extrinsic motivator - meeting a class requirement - may therefore pave the way for students to realize intrinsic motivators for service participation. The most frequently cited responses from participants are helping to make a difference in people's lives, having the opportunity to engage with others, being passionate about the cause, and gaining different experiences. Staff responses frequently reference terms such as "intrinsic" and "values" at the root of students' motivation to participate in service, though they also often mention the benefit of obtaining tangible skills learned from service participation. The total frequencies of each coded motivator and the number of interview and/or observation transcripts with that coded motivator are listed in Table 1.

The distribution of motivations differs by the type of service and type of events students discuss, and staff recognize the distribution differences as trends revealed through their observation of volunteer involvement. Most students participate in both episodic and sustainable service with different organizations and had participated in each type of 
event: direct, fundraising, and awareness. Events of each type were observed.

Interviews with students revealed that episodic service is most frequently associated with students wanting to help others, while gaining different experiences, having passion about the cause, and engaging socially with others were secondary motivators. However, observations of episodic service events reveal a slightly different distribution. Episodic service involvement is readily accessible to students; the signup process is easily found on social media and online and dayof involvement sign-ups are encouraged. Consequently, these events are viewed by participants as perfect opportunities for social engagement; student volunteers can see which other appears that direct service is equally easy and important to execute for episodic and sustainable events.

\section{Service in a Liberal Arts Education}

Student interviewees agree that, whether sustainable or episodic, service as part of a liberal arts education is necessary to develop graduates with a widened lens of the world. No matter the motivation for service participation, GC emphasizes service as a transformative experience for students that prepares them for their profession and life after graduation. In fact, GC's (2019) mission puts a heavy emphasis on the importance of a liberal arts education in ways that support service; it specifically highlights "taking learning beyond the

Table 2. Perception of University Involvement with Service

\begin{tabular}{|l|lc}
\hline Perception & Total frequency & Transcript frequency \\
\hline High involvement & 18 & 9 \\
\hline Moderate involvement & 6 & 4 \\
\hline Low involvement & 17 & 9 \\
\hline
\end{tabular}

students are attending the event. As a result, observing volunteers who clearly know each other engage in personal conversations is not unexpected.

Although students' motivations to participate in sustainable service are the same as episodic service, the motivations are referenced in different frequencies. Students participating in sustainable service reference wanting to help others most often, followed by having passion about the cause, gaining different experiences, and engaging socially with others. Social engagement, however, is more often described as a result of participating rather than a reason. Observations reflect this trend; volunteers appear to either already have a relationship through the organization or attempt to build new relationships through the shared experience of helping others, rather than students attending the event because they know each other.

It is likely the overlapping motivations for each type of service are a result of the overlapping constructs of sustainable versus episodic service, as seen when comparing the types of service events. Over half of the interviewees participate in some type of fundraising, though it is difficult to determine whether this type of event should be considered sustainable or episodic. Though most fundraising events are episodic, the goal of fundraising in most cases is to provide resources with which an organization can sustainably meet a need. Less than half of the interviewees participated in awareness events, but the episodic versus sustainable debate is also present. People and organizations are passionate about a sustainable cause but are executing episodic awareness events to support that cause. Also, some degree of direct service is necessary to execute each type of event. It is likely that all participants claimed to have participated in direct service for this reason. Preparing for and orchestrating the events could be considered direct service regardless of whether the goal of the event was to raise funds, raise awareness, or perform physical labor to reach a goal. It traditional classroom" and "developing civic skills that enable graduates to thrive in a diverse global society" and claims that "through service, GC enriches the lives of students and their local and global communities". For these reasons, the GIVE Center provides a unique and beneficial experience that is valued both by GC as a whole and by students who volunteer. In fact, student interview participants believe that the GIVE Center provides a positive service environment to reach the GC mission and adequately assists student-led service organizations. The GIVE Center provides everything from minimal, logistic involvement to hosting, which includes providing supplies and volunteers for some events. However, perceptions varied among students and staff about whether Georgia College as a whole is already effective, not very effective, or is trying to be more effective at incorporating and encouraging service throughout campus. The total frequencies of each coded perception and the number of interview and/or observation transcripts with that coded perception are listed in Table 2.

Most student interviewees believe service as part of a liberal arts education provides a means of expanding one's perspective, and the purpose of service is to expose students to conditions and populations they may not have been otherwise. Georgia College students' families have a mean annual income of over \$100,000 (Gregor Aisch, 2017) and likely have little experience with those in poverty or poor living conditions. Many student interviewees recognize this, and some participants reference the lack of diversity in terms of the student population, though there was no specific reference to what type of diversity. However, the term "diverse" is also used in relation to the service experiences and types of service students participate in, as well as to describe the experiences needed for jobs that service participation provides. Though only some students referenced the specific language of "diverse" and "diversity," those who used it did so more than once throughout their interviews. The total frequencies of each 
coded perception and the number of interview and/or observation transcripts with that coded perception are listed in Table 3.

Staff interviewees share similar perceptions to students mentioned literature, service participation in college seems to provide similar, positive results regardless of whether it is mandatory or voluntary, though the reasons for participating are different.

Table 3. Perception of Service in a Liberal Arts Education

\begin{tabular}{|l|l|l|}
\hline Perception & Total frequency & Transcript frequency \\
\hline Is needed & 29 & 9 \\
\hline Expands perspectives & 20 & 9 \\
\hline Promotes "Diversity" & 7 & 3 \\
\hline
\end{tabular}

with a few differences. In contrast to students, staff describe service as an opportunity to not only expand one's perspective but also to increase students' knowledge, resume experience, and other skills that will be used in their profession. They emphasize the importance of a liberal arts education in the creation of citizens who are engaged and are leaders in their community, as mentioned in the Georgia College mission. One participant on the student affairs staff explained that student volunteers do not realize they gain this advantage while participating in service.

\section{Discussion}

\section{Motivations for Service Participation}

The results of this research support the findings and theories in previous literature. For example, participants' motivations for volunteering provided some overlap with previous findings. The guilt of privilege and a felt responsibility or obligation towards other people is mentioned by students, as well as engaging with service recipients and seeing their happiness. Additionally, students recognize service involvement as an easy opportunity to expand their resume. One of the largest overlaps is the drive to help people and make a difference in social issues about which they were passionate.

However, there are additional motivations that were not listed in the previous literature. Social engagement with other student volunteers, for example, is a predominant motivator for these participants, though more often it is a reason for episodic volunteering and a result of sustainable volunteering. Additionally, doing service for the purpose of gaining new experiences, whether for the purpose of being experienced in their future career or because there were easy opportunities to gain these experiences, is a new concept. This could be explained because Georgia College is a liberal arts school that encourages perspective expansion or because the Georgia College GIVE Center caters an assortment of service events specifically to students. More studies at other liberal arts institutions that prioritize broadening perspectives could confirm this hypothesis.

Other research suggests that mandatory service, meaning it is required by classes, academic programs, Greek life, scholarships or awards, has a positive effect on students after college. Student participants in this study, however, do not frequently associate these extrinsic motivators with service. When they do, it is only associated with their initial service experiences. Participants more frequently reference intrinsic motivators, such as their feelings, beliefs, and values, as reasons they continue to participate in service, demonstrating that they believe their participation is entirely voluntary. According to students in this study and findings in previously
There were not considerable differences in this study between students' motivations to participate in episodic versus sustainable service, and this pattern of overlap suggests that the difference in student participation is related to other factors. Research suggests, for example, that students who participate episodically may be overextended in other aspects of their life but still want to serve others in the time they have available, while students who participate sustainably may focus fully on service because it provides experience they need for a future career. This study supports these findings. Students who participate sustainably stated they want to continue serving others, and those who participate episodically were usually involved in another aspect of campus life or more than one service organization.

\section{Service in a Liberal Arts Education}

As suggested by previous literature and supported by this research, a liberal arts education provides a method to create engaged citizens throughout the college experience and after graduation. Many students who participate in service want to pursue a service-oriented career field or continue to volunteer with nonprofits relevant to the organizational causes they work with as students in order to continue to make a difference. These same students overwhelmingly feel that service participation not only benefits their overall college experience but also enriches their liberal arts education. Student respondents stated that participating in service makes them feel more outgoing, more passionate about the causes with which they volunteered, and more prepared for life after college. It is clear that extracurricular service in liberal arts is received positively by this school population. However, there are mixed opinions regarding co-curricular service. Some participants believe it is a gateway into voluntary service, while others believe it drives students away from service. The most prominent argument, however, is that those who do not want to pursue service will avoid it, and those who want to pursue service will pursue it, regardless of its requirement.

\section{Procedures}

Semi-structured interviewing was conducted with students who actively pursue service opportunities and Student Affairs staff at Georgia College who are interested in the concept of service and frequently observe students' participation. For the purposes of this research, "active" applies to any person who, of their own volition, chooses to pursue and participate in community service activities. Semi-structured interviewing allowed for the flexibility required to accommodate the diverse array of service activities students pursue. The interview protocol contained some specific questions about perceptions of community and community 
service, as well as Georgia College's infrastructure to support student service (e.g. GIVE Center) as a liberal arts institution. However, the protocol also allowed participants to discuss their unique experiences and motivations for service participation in a narrative form while allowing the researcher to probe different areas according to what was discussed by the interviewee. Interview protocols for student and staff are listed in Appendices A and B.

Ten interview participants were recruited through purposive sampling, utilizing the researcher's resources and contacts through the GIVE Center to aid in the selection of possible participants. After interviewees consented, the interviews were audio recorded and later transcribed by the researcher. Interviewees were not asked to state their name for the recordings, and the only possible identifying information collected was in regard to their service participation or leadership roles. Interviewees chose the location of the interview, within reason, and indirectly determined the length of the interview through their willingness to expand on their experiences. Interviews mainly occurred on or near the university's campus, and the length of the interviews ranged from nine minutes to thirty-six minutes.

Participant observation was utilized at both episodic service events and sustainable service opportunities to gain a holistic student service experience and provide other insight to the culture of volunteering through students' interactions and nonverbal cues. The goal was to participate in service opportunities the researcher has never attended before. (The researcher's participation in service opportunities, especially with student organizations, was not uncomfortable for research participants since the researcher is already an active participant in service opportunities.) The location of the event, number of students in attendance, and a general summary of what occurred at each event was recorded for all six events. The observation opportunities were chosen as suggested by the GIVE Center and interview participants to further understand the student volunteer experience.

Transcriptions of each interview and service event were analyzed utilizing QDA Minor software, in which sections of the transcripts were classified into codes relating to all aspects of the research, including participant attributes, service event information, motivations for service involvement, and perceptions of service in relation to Georgia College. The subcodes of these areas revealed the most significant information through specific phrases or words used or implied most frequently in the interviews or observed at service events.

\section{Acknowledgements}

Acknowledgements are given to Dr. Amanda Reinke (research advisor and mentor to the researcher), the interview participants, the service organizations that allowed observation at their events, Georgia College, and the GC GIVE Center. The researcher acknowledges a possible conflict of interest with the GIVE Center due to her personal voluntary service involvement and employment with the office while conducting this research.

\section{References}

Association of American Colleges \& Universities. (2019). Retrieved from https://www.aacu.org/

Dote, L., Cramer, K., Dietz, N., \& Grimm, R. (2006). College Students Helping America (pp. 1-23, Rep.). Washington, DC: Corporation for National and Community Service.

Fenzel, L. M., \& Peyrot, M. (2005). Comparing College Community Participation and Future Service Behaviors and Attitudes. Michigan Journal of Community Service Learning, 12(1), 23-31. Retrieved from: https://gcsu.idm.oclc.org/login?url=http://search.ebs cohost.com/login.aspx?direct $=$ true $\& \mathrm{db}=$ eric $\& \mathrm{AN}=$ EJ848479\&site $=$ eds-live\&scope $=$ site Georgia College. (2019). Retrieved from https://www.gcsu.edu/

Gregor Aisch, L. B. (2017, January 18). Economic diversity and student outcomes at Georgia College \& State University. New York Times. Retrieved from https://www.nytimes.com/interactive/projects/colleg e-mobility/georgia-college-state-university

Heffner, G. G., Curry, J. M., \& Beversluis, C. D. (2006). Transforming Liberal Arts Education through Engaged Scholarship. Journal of Higher Education Outreach and Engagement, 11(1), 119-132. Retrieved from: https://gcsu.idm.oclc.org/login?url=http://search.ebs cohost.com/login.aspx?direct $=$ true $\& \mathrm{db}=$ eric $\& \mathrm{AN}=$ EJ1093027\&site $=$ eds-live\&scope $=$ site

Jones, K., Blinkhorn, L. M., Schumann, S.-A., \& Reddy, S. T. (2014). Promoting sustainable community service in the 4th year of medical school: A longitudinal service-learning elective. Teaching and Learning in Medicine, 26(3), 296-303. DOI: 10.1080/10401334.2014.911698

McCunney, D. (2017). Shaped by Campus Culture: Intersections Between Transformative Learning, Civic Engagement, and Institutional Mission. Journal of Higher Education Outreach and Engagement, 21(3), 61-67.

Sherman, G. (2016). Service Learning in Light of Emmanuel Levinas. Studies in Philosophy \& Education, 35(5), 477. DOI: $10.1007 / \mathrm{s} 11217-015-94930$

White-Farnham, J., \& Meyer, J. (2014). Who is Being Served by Academic Service-Learning? Distinctions from the Public Liberal Arts College. Currents in Teaching \& Learning, 7(1), 80-88. Retrieved from https://gcsu.idm.oclc.org/login?url=http://search.ebs cohost.com/login.aspx?direct=true $\& \mathrm{db}=$ eue $\& A N=1$ 01595966\&site $=$ eds-live \&scope $=$ site 